\title{
Characterization and visualization of RNA secondary structure Boltzmann ensemble via information theory
}

\author{
Luan Lin ${ }^{1 \dagger}$, Wilson H. McKerrow ${ }^{2 \dagger} \mathbb{D}$, Bryce Richards ${ }^{3}$, Chukiat Phonsom ${ }^{4}$ and Charles E. Lawrence $2^{*}$
}

\begin{abstract}
Background: The nearest neighbor model and associated dynamic programming algorithms allow for the efficient estimation of the RNA secondary structure Boltzmann ensemble. However because a given RNA secondary structure only contains a fraction of the possible helices that could form from a given sequence, the Boltzmann ensemble is multimodal. Several methods exist for clustering structures and finding those modes. However less focus is given to exploring the underlying reasons for this multimodality: the presence of conflicting basepairs. Information theory, or more specifically mutual information, provides a method to identify those basepairs that are key to the secondary structure.

Results: To this end we find most informative basepairs and visualize the effect of these basepairs on the secondary structure. Knowing whether a most informative basepair is present tells us not only the status of the particular pair but also provides a large amount of information about which other pairs are present or not present. We find that a few basepairs account for a large amount of the structural uncertainty. The identification of these pairs indicates small changes to sequence or stability that will have a large effect on structure.

Conclusion: We provide a novel algorithm that uses mutual information to identify the key basepairs that lead to a multimodal Boltzmann distribution. We then visualize the effect of these pairs on the overall Boltzmann ensemble.
\end{abstract}

Keywords: RNA, RNA secondary structure, Nearest neighbor model, Information theory, Mutual information

\section{Background}

RNA plays an important role in many biological processes, and next generation sequencing technologies have revealed a large number of novel non-coding RNA transcripts whose roles in biological processes are only beginning to be understood. Because the structure of macromolecules is often key to their function, the discovery of RNA structure has become increasingly important. While much progress has been made in the experimental determination of RNA structure, the disparity between RNA structure and sequence has continued to grow [1]. Thus computational tools that illuminate the physics of RNA structure are as important as ever.

${ }^{*}$ Correspondence: charles_lawrence@brown.edu

${ }^{\dagger}$ Equal contributors

2Division of Applied Mathematics, Brown University, 02912 Providence, RI, USA Full list of author information is available at the end of the article
Because secondary structure (SS) provides by far the largest contribution to the overall stability of an RNA molecule and precedes 3-D contact formation in the folding process, algorithms for the prediction of RNA SS continue to be an important component of structural prediction [2]. RNA SS algorithms have been developed for the prediction of structure from multiple related sequences $[3,4]$ and for SS prediction from a single RNA sequence. Here we focus on the latter class. The most popular RNA SS algorithms use recursive dynamic programming methods based on nearest neighbor energy calculations: to find the minimum free energy (MFE) structure [5-7]; to find the partition function [8]; to sample from the Boltzmann weighted ensemble [9] and to predict structures from the Boltzmann ensemble $[10,11]$. 
However despite the progress that has been made, prediction of RNA SS from a single sequence remains challenging, especially for longer sequences. Many RNA structures are bistable, forming different structures in different contexts. Others form pseudoknots: structural features that are excluded from standard RNA secondary structure prediction methods. But even for sequences with a single, known native structure containing no pseudoknots, the Boltzmann distribution is rarely unimodal. This has led to efforts to find clusters of structures when no single representative structure exists. Methods include standard clustering algorithms [11-13] and strategies tailored to RNA SS: RNAshapes [14-16] finds structures that share a common "shape", and Rogers et al. [17] group structures that share common helices in a process called "profiling". Both of these strategies simplify the RNA folding problem by abstracting from individual basepairs to the helices that define RNA SS. While grouping structures based on common features does allow for a simplified description, such methods do not provide insight into the underlying features - conflicting basepairs - that drive multimodality in the Boltzmann distribution. Identifying these conflicting pairs will provide insight into how these alternate structures interact.

Recent work on so called "riboSNitches" has shown that many SNPs in noncoding sequences have wide ranging and potentially disease inducing effects on RNA structure [18-20]. The presence of disease associated variants in noncoding regions highlights the need to understand the relationship between sequence variation and RNA SS [20]. However predicting potential riboSNitches remains difficult [21]. Finding a few basepairs that are key to the secondary structure indicates that a mutation preventing the formation of one of these pairs will send the structure into an alternate conformation with potentially harmful effect. Furthermore, for some RNAs, such as viral genomes, alternative structures are necessary for proper function [22]. Even if the alternate conformations differ widely, the differences can often be reduced to the presence or absence of a few pairs. Finding these pairs provides an insight into how the transition between conformations is controlled. Finally, the rapid folding of an RNA into its native structure requires avoiding kinetic traps [23]. Thus, identifying key conflicting basepairs indicates which pairs must be avoided and which pairs must form in order for an RNA to fold quickly.

We employ information theory, or more specifically mutual information to find these key conflicting basepairs. Information entropy has been used to measure the complexity of the Boltzmann ensemble [24, 25], and the mutual information between aligned sequences has been used to construct a consensus sequence [26, 27]. However less focus has been given to the mutual information between the basepairs of a single RNA molecule.
Using the nearest neighbor model (excluding pseudoknots), as implemented in the RNAstructure package [28], our algorithm finds the basepairs that provide the most information about other basepairs: the most informative base pairs (MIBPs). We then visualize the effect of these pairs by plotting the marginal basepairing probabilities conditioned on the presence or absence of the MIBPs.

\section{Methods}

\section{Nearest neighbor model}

An RNA secondary structure (SS) is a string of bases (A, $\mathrm{C}, \mathrm{G}$, or $\mathrm{U}$ ), called the sequence, together with a set of basepairs between non-adjacent letters. Basepairs are two element sets, where $\{i, j\}$ denotes a pair between the $i^{t h}$ and $j^{\text {th }}$ bases. For $1 \leq i<j \leq n, X_{i j}$ is a random variable that is 1 when the $\{i, j\}$ pair is present and 0 when it is not. Only Watson-Crick (A-U, G-C) and wobble (G-U) pairs are considered. The space of allowable secondary structures is constrained by the following two requirements:

1. (No triples): $\sum_{j} X_{i j} \leq 1$ for all $i$.

2. (No pseudoknots): $X_{i j}+X_{k l} \leq 1$ for all $i<k<j<l$.

If these requirements prevent two basepairs from existing simultaneously, we say that they conflict. Namely $\{i, j\}$ and $\{k, l\}$ conflict if $i$ or $j$ equals $k$ or $l$, if $i<k<j<l$, or if $k<i<l<j$. If we draw basepairs as lines through a circle as in Fig. 1, conflicting pairs intersect on or inside the circle.

The free energy of a structure is given by experimentally derived parameters detailing the stability of

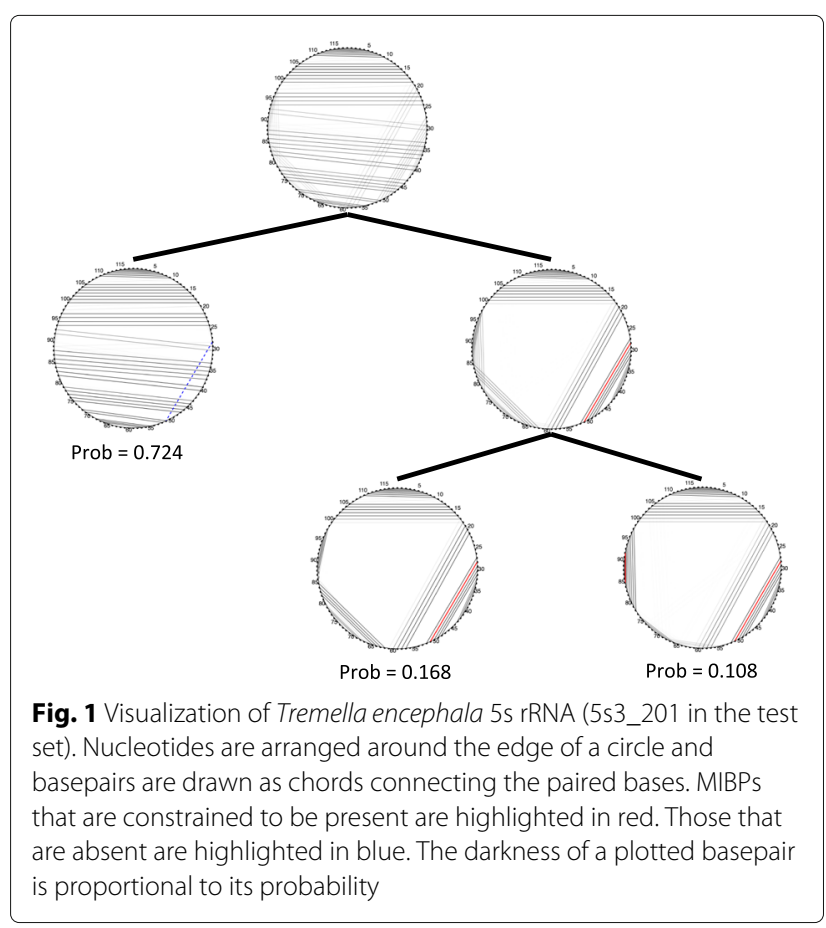


various configurations of helices, loops and bulges. The Boltzmann probability is then proportional to the exponent of the negative free energy. In this paper we use the RNAstructure software [28] to calculate Boltzmann probabilities and to sample structures directly from this distribution.

\section{Most informative basepair}

We equate the complexity (or simplicity) of a distribution with how unsure we are about the value of the corresponding random variable. The uncertainty in a basepair $\{i, j\}$ is measured using information entropy:

$$
H\left[X_{i j}\right]=-p_{i j} \log _{2} p_{i j}-\left(1-p_{i j}\right) \log _{2}\left(1-p_{i j}\right)
$$

where $p_{i j}=P\left(X_{i j}=1\right), 0 \log 0=0$ and the units of $H$ are in bits. When we are less sure about a basepair, $p_{i j}$ is closer to $1 / 2$ and $H\left[X_{i j}\right]$ is larger. Conversely when we are more sure about a basepair, $p_{i j}$ is closer to 0 or 1 and $H\left[X_{i j}\right]$ is smaller.

Now if we condition on another basepair $X_{k l}$, we have two conditional distributions: $P\left(X_{i j} \mid X_{k l}=1\right)$ and $P\left(X_{i j} \mid X_{k l}=0\right)$, each with corresponding entropies: $H\left[X_{i j} \mid X_{k l}=1\right]$ and $H\left[X_{i j} \mid X_{k l}=0\right]$. The conditional entropy is defined to be

$$
H\left[X_{i j} \mid X_{k l}\right]=\left(1-p_{k l}\right) H\left[X_{i j} \mid X_{k l}=0\right]+p_{k l} H\left[X_{i j} \mid X_{k l}=1\right]
$$

and is the average uncertainty in $X_{i j}$ after we learn the value of $X_{k l}$. Therefore the amount of information that $X_{k l}$ tells us about $X_{i j}$ is

$$
I\left(X_{i j} ; X_{k l}\right)=H\left[X_{i j}\right]-H\left[X_{i j} \mid X_{k l}\right]
$$

This value is referred to as the mutual information between $X_{i j}$ and $X_{k l}$ and it is symmetric [29]. By Eqs. 2 and 3, on average the distribution of $X_{i j}$ conditioned on $X_{k l}$ is $I\left(X_{i j} ; X_{k l}\right)$ bits simpler than the unconditioned distribution. We can then measure the amount of information that a basepair provides about the rest of the secondary structure by adding up its mutual information with each other basepair. We can then condition on the basepair that has the greatest sum of mutual information to get a less complex conditional distribution. We call this basepair the most informative basepair:

Definition 1 The most informative basepair (MIBP) is the basepair that has the largest sum of mutual information:

$$
\operatorname{MIBP}=\underset{k l}{\operatorname{argmax}} \sum_{i j} I\left(X_{i j} ; X_{k l}\right)
$$

Calculating mutual information requires the joint probability of every pair of basepairs, a computationally intensive task. However we can quickly estimate the mutual information from sampled structures. Structures can be sampled from the Boltzmann ensemble for a sequence of length $n$ in $\mathcal{O}\left(n^{3}\right)$ time using RNAstructure or a similar tool. We can find the MIBP from sampled structures as follows:

\section{Algorithm 1}

1. Get 1000 samples from the Boltzmann distribution

2. Considering only pairs that appear in at least 10 and less than 990 samples, estimate the joint probability for each pair of pairs.

3. Use the estimates to calculate mutual information for each pair of basepairs.

4. Sum the mutual information of each basepair.

5. Find the basepair that has the greatest sum.

Basepairs that appear in fewer than 10 or more than 990 samples will have low entropy and so will not make a significant contribution to mutual information. Thus we can improve computational efficiency without sacrificing accuracy by ignoring them. In general we find that 1000 samples is enough to get an accurate estimate of base pairing probabilities.

\section{Tree based clustering}

We greedily employ the MIBP algorithm to build a binary tree that clusters structures based on the presence of MIBPs. We first split the space into a cluster that includes the MIBP and one that does not. We then find the conditional MIBP in each of the clusters and split those clusters in two. We continue this process until the product of the cluster probability and estimated mutual information falls below 2 bits. The algorithm creates a binary tree of
Algorithm 2
1. Label ensemble cluster".
2. For all clusters $x$ :
(a) Calculate MIBP
(b) If $P(x) *$ MI $>2$ bits: Split $x$ into cluster $x 0$ without MIBP and cluster $x 1$ with MIBP.

3. Repeat step 2 until no new clusters are made.

nested clusters, where each branch corresponds to conditioning on the presence or absence of a particular MIBP. The leaves of this tree are then an exhaustive set of clusters. An html file is created that draws the tree and plots the marginal basepairing probabilities at each node. See the "Results" section for examples. Algorithms that use mutual information to create binary trees, such as ID3 
and C4.5 [30], are used widely in classification problems. This algorithm employs a similar concept, but it uses the mutual information between basepairs as there is no natural labelling for RNA secondary structures as would be the case in a classification problem.

\section{Conflicting basepairs and other cluster calculations}

Once we have found MIBPs and divided the space, we can examine the individual clusters. First we look for basepairs that conflict with the MIBP. For each MIBP split, we first find the basepair that conflicts with the MIBP and is most probable. Because the MIBP and the conflicting pair cannot be present in the same structure, the most probable conflicting pair is also the conflicting basepair that has the most pairwise mutual information with the MIBP (see "Additional methods" section). We then continue in a greedy fashion, repeatedly finding the most probable basepair that conflicts with the MIBP and all previous conflicting pairs. We stop once we have found a total of five conflicting pairs. When conflicting pairs are present, they provide an explanation for the presence of divergent clusters.

We can also use RNAstructure to calculate the marginal probability of each basepair in each cluster and use that information to calculate the conditional entropy in each cluster. Finally, we can calculate the number of structures in each cluster by setting the free energy, $E(x)$, equal to 0 for all structures $x$ and then calculating the partition function. Note that RNAstructure counts structures with the same basepairs but different coaxial stacking separately.

\section{Results}

To test our algorithm, we used a set of sequences and corresponding native structures provided to us by David Mathews. This data is used by the Mathews group to test the RNAstructure software package. The sequences are compiled from the following publications: [31-40]. The test set includes sequences from ten families whose secondary structures have been verified by comparative analysis: 5s, 16s and 23s rRNA, group 1 and 2 introns, RNAse P (RNAp), signal recognition particle (SRP), telomerase, tmRNA and tRNA. The $16 \mathrm{~s}$ and $23 \mathrm{~s}$ rRNA sequences were divided into four and six folding domains respectively [32, 33 ] to make the computation more tractable. For $5 \mathrm{~s}, 16 \mathrm{~s}$, SRP, telomerase, tmRNA and tRNA, we considered only 10 randomly selected sequences from each family as the test set included a large number of sequences from these families. A list of all sequences considered can be found at the visualization site described in the next section.

\section{Visualizations}

Visualizations of the Boltzmann ensembles of these sequences can be found at http://ccmbweb.ccv.brown. edu/wmckerro/MIBP/Visualizations should be viewed in the firefox browser. Longer sequences may be slow to load. The visualizations draw the binary tree described in the "Methods" section. Clicking on a node in the tree reveals the conditional probability of each basepair in the corresponding cluster, showing how the presence or absence of MIBPs affects the structure. Figure 1 shows the circle diagrams arranged in a tree for an example RNA molecule.

\section{Entropy reduction}

To see how adding new clusters affects the conditional entropy, we ran our algorithm for 100 steps on one sequence from each family, yielding 101 clusters for each sequence. As a function of the number of clusters, the entropy closely follows a power law with an exponent that varies from -0.1 for the Chinchilla brevicaudata telomerase (AF221937.99-545 in the test set) and trna sequences to - 0.4 for the Clostridium perfringens tmRNA sequence (Clos.perf._CP000246_1-361). (See Fig. 2.)

We also test how the default 2 bit cutoff (see "Methods" section) affects the conditional entropy. Across all the seqeunces tested, the 2 bit cutoff yielded an average of 3.4 clusters with average entropy reduction of nearly

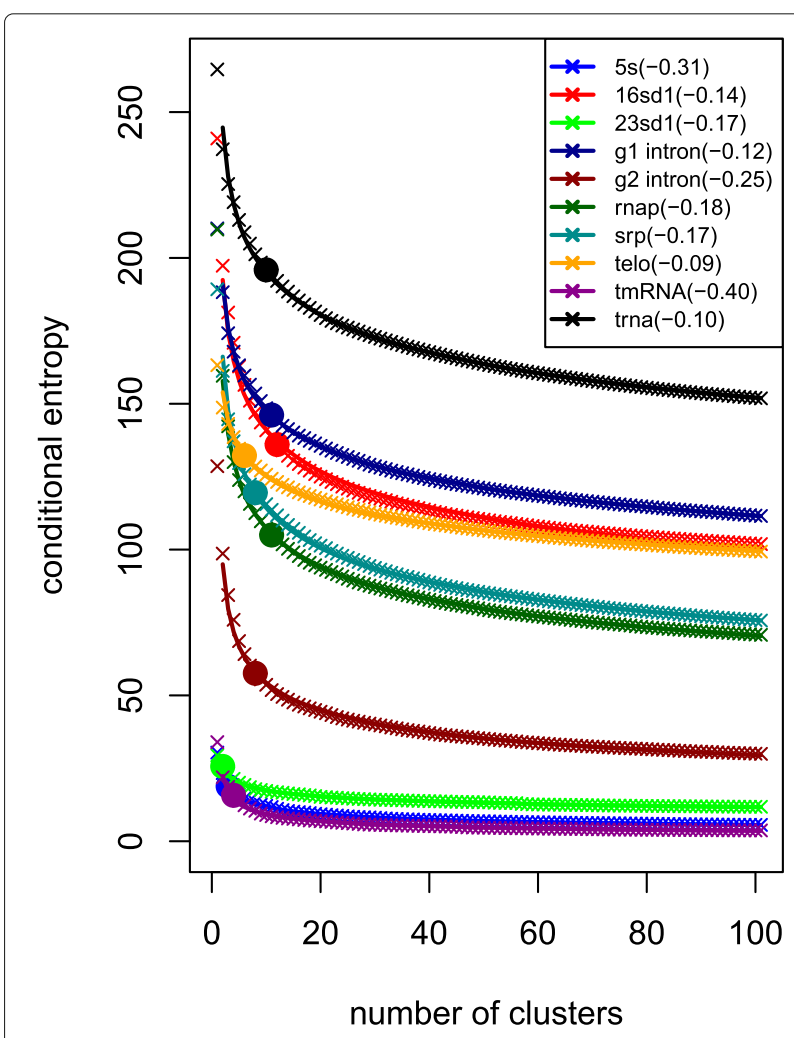

Fig. 2 Entropy as a function of number of clusters for one sequence from each of the ten families. Power law functions of the form $y=a x^{b}$ are estimated by linear least squares regression after log-log transform and plotted as lines. The value of $b$ is given parenthetically. The two bit cutoff is highlighted by a filled circle 
a half (See Fig. 3). This reduction is slightly larger than would be predicted by the power law because the first couple splits often provide greater entropy reduction. It would be possible to use a smaller cutoff, yielding more clusters, but the power law functions indicate that this would likely yield only a modest decrease in ensemble entropy.

\section{Entropy constraints and number of structures}

Constraining basepairs with low entropy excludes most of the possible structures, but retains most of the probability mass. This is consistent with concentration of measure phenomena often seen in high dimensional probability distributions [41]. Basepairs with entropy less than 0.002 bits were constrained to be unpaired if they have probability near 0 and paired if they have probability near 1. For every sequence, the entropy constraints removed less than $5 \%$ of the probability mass but resulted in about a one fourth reduction in the orders of magnitude for the total number of possible structures. For a short sequence such as Spirocodon saltatrix 5s rRNA (5s3_220) this means a reduction of 8 orders of magnitude from $4 \times 10^{31}$ to $6 \times 10^{23}$. However for a longer sequence such as a Saccharomyces cerevisiae group II intron (ya1) the entropy constraints result in a reduction of almost 50 orders of magnitude: from $1 \times 10^{172}$ to $9 \times 10^{126}$ (see Fig. 4).

\section{Cluster with native structure}

Consistent with previous observations [11] the fact that a cluster contains more probability does not necessarily

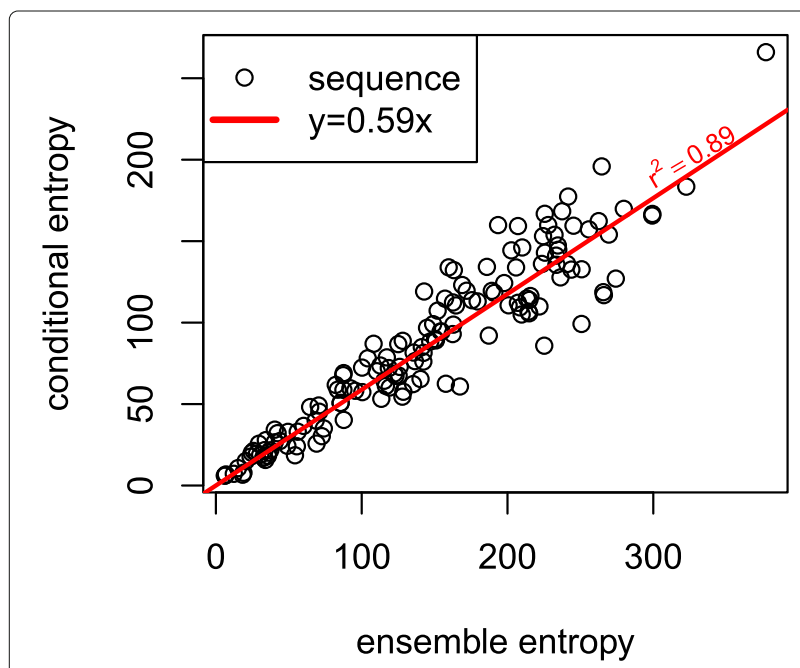

Fig. 3 Ensemble entropy vs conditional entropy. Running the MIBP algorithm with a 2 bit cutoff yields an entropy reductions of nearly a half. For example the Chlamydomonas 5s rRNA (5s_13 in the test set) has an ensemble entropy of 49 bits, but after conditioning on the MIBPs, only 25.5 bits of uncertainty remains. Each point represents one of the sequences from one of the ten families described at the beginning of the results section

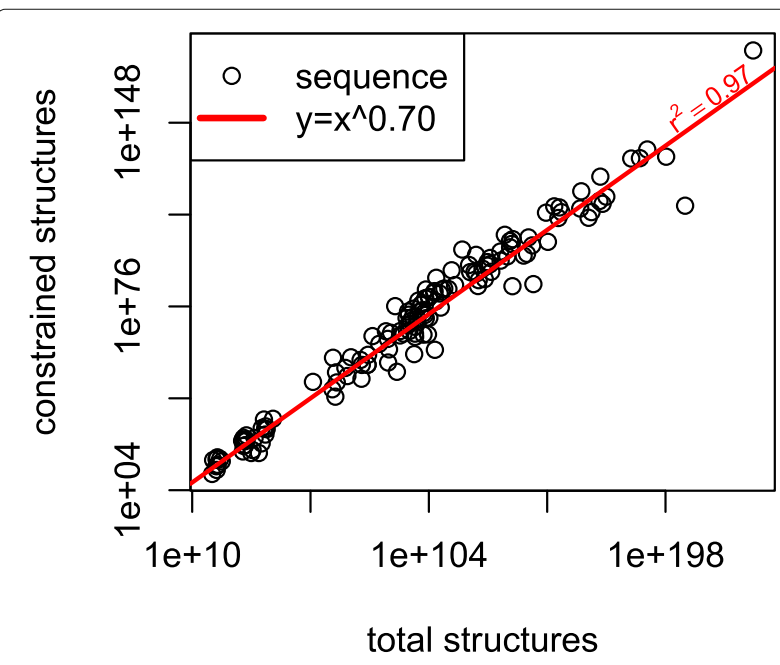

Fig. 4 Number of structures before and after basepairs with entropy less than 0.002 bits are constrained. Constraining basepairs reduces the orders of magnitude by about one fourth. Each point represents one of the sequences from one of the ten families described at the beginning of the results section

mean that it will contain the native structure. In fact, for the sequences tested, the probability of the native cluster is not significantly larger than the probability of a cluster chosen uniformly at random (permutation $p$-value $=$ 0.47). This is likely due to the fact that secondary structure prediction algorithms struggle to provide accurate predictions for some of the longer sequences considered. If we rerun the analysis on the $3095 \mathrm{~s}$ RNA sequences in the RNAstructure test set, we find that the average native cluster size is $41.5 \%$ - significantly higher than the mean random cluster size of $37.0 \%$ (permutation $p$-value $=0.02$ ). However it is still far short of the mean expected cluster size of $51.9 \%$. This indicates that, at least for smaller sequences, the native structure is more likely to be found in a higher probability cluster, but that it less likely to be found in such a cluster than the Boltzmann ensemble indicates. Permutation tests were done in $\mathrm{R}$ using the coin package with 10,000 samples.

\section{Conflicting basepairs}

We find that many MIBPs are part of a pair of conflicting basepairs, but we also find that in many cases the MIBP is part of a set of more than two mutually conflicting basepairs. Each pair in the set of mutually conflicting pairs is somewhat probable on its own, but due to the no pseudoknots and no triples constraints, only one can be present in a given structure.

For $40 \%$ of MIBPs, the MIBP represents a binary choice between two basepairs. In such cases there is a conflicting base that is present in at least $90 \%$ of sampled structures that do not include the MIBP. In other cases the MIBP is one choice among a set of mutually conflicting pairs. For 
$84 \%$ of MIBPs, $90 \%$ of samples that do not include the MIBP include one member of a set of up to five basepairs that conflict with each other and the MIBP.

\section{Mutations to the MIBP nucleotides}

In this subsection we consider a 118 nucleotide $5 \mathrm{~s}$ rRNA from the freshwater alga Hydrurus foetidus (5s3_71 in the test set). The MIBP algorithm finds one most informative basepair - $(17,61)$ - dividing the Boltzmann space into two classes. $82 \%$ of structures that do not contain the MIBP contain the conflicting pair $(29,107)$. This implies that mutating the sequence so that one of these two basepairs cannot form would bias the structure to fall into one class over the other. Indeed, editing the 17th nucleotide from a $\mathrm{C}$ to a $\mathrm{A}$ yields a Boltzmann distribution that is similar to conditioning on the absence of the MIBP. Editing the 29th position from a $\mathrm{C}$ to a $\mathrm{G}$ yields a structure that is similar to conditioning on the presence of the MIBP. However a different set of basepairs constitute one of the helices (see Fig. 5).

\section{Mutual information and RiboSNitches}

Woods and Laederach [42] use SHAPE data to classify mutations into three categories based on whether they cause (i) "no differences or small differences", (ii) "local differences", or (iii) "global differences" to the RNA secondary structure. We focus on one of the sequences considered by Woods and Laedarach: a 16s rRNA 4 way junction (16SFWJ_1M7_0001 in RMDB: https://rmdb. stanford.edu/). While the ends of the $\operatorname{MIBP}(146,216)$ are not mutated, nearby positions that are likely to form a helix with the MIBP are mutated: a $G$ to $C$ mutation at position 125 causes local differences, and $\mathrm{C}$ to $\mathrm{G}$ mutations at positions 214 and 221 causes global differences. Most of the mutations considered (74\%) cause little or no difference to the RNA SS. The third mutation that affects global change, a $\mathrm{G}$ to $\mathrm{C}$ mutation at position 177 , forms the conflicting pair for one of the three additional MIBPs found with the standard 2 bit cutoff.

We also compare the mutation category to the maximum sum of mutual information for a basepair originating
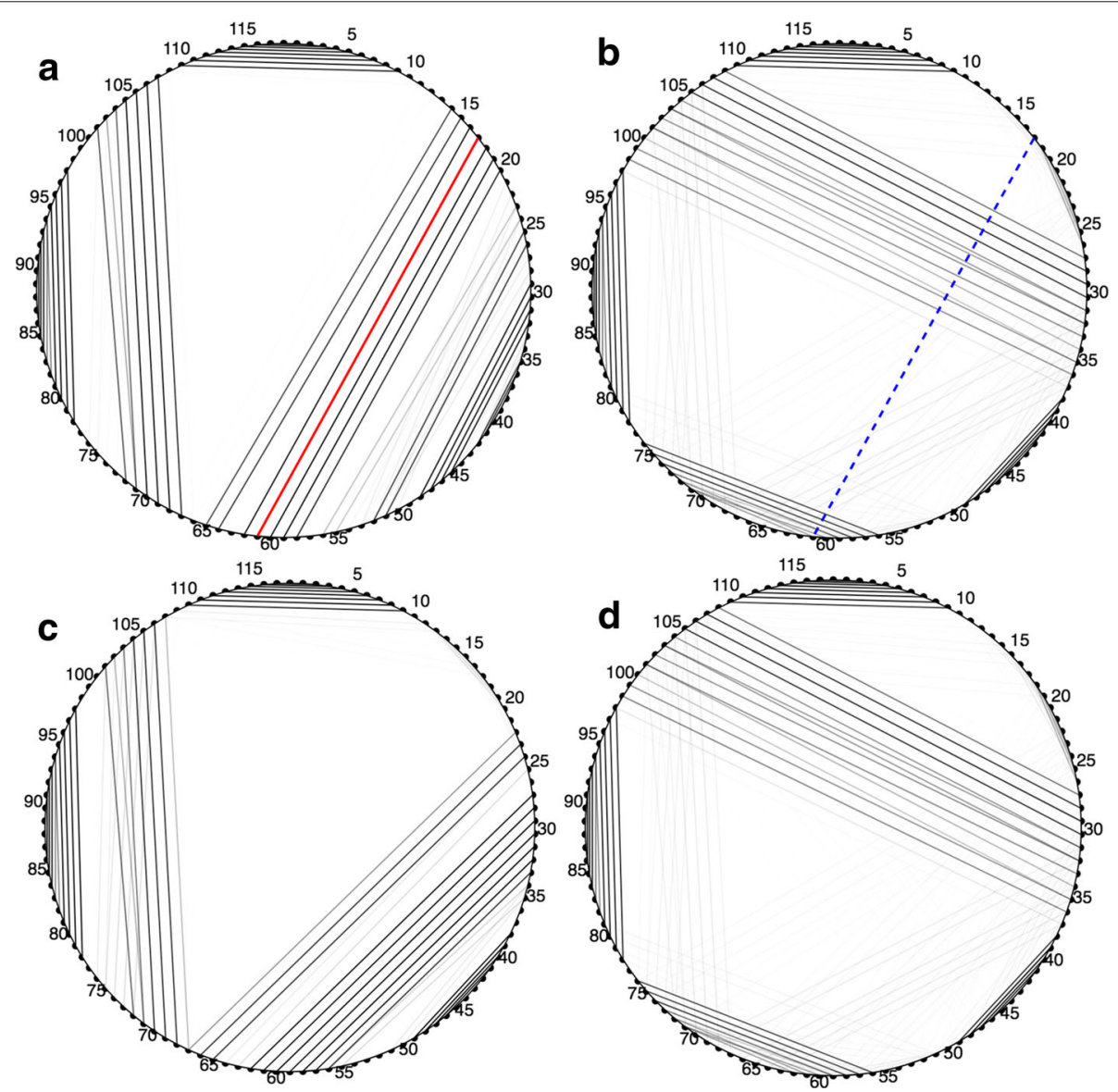

Fig. 5 Mutating the ends of the MIBP or conflicting pair has a large effect on the resulting RNA SS. a Basepair probabilities conditioned on the presence of MIBP. b Basepair probabilities conditioned on the absence of MIBP. c Basepair probabilities when conflicting pair is mutated. $\mathbf{d}$ Basepair probabilities when MIBP is mutated. 5s rRNA from the freshwater alga Hydrurus foetidus (5s3_71 in the test set) is the sequence considered 
from the mutated position. The mean mutual information for mutations that cause global changes in RNA SS (9.85 bits) is greater than the mean MI for positions local changes $(7.36$ bits) and much greater than the mean MI for positions that cause little or no change (3.06 bits). Figure 6 shows the mutual information at all the mutated positions. The html visualization for this molecule can be accessed at: http://ccmbweb.ccv.brown. edu/wmckerro/MIBP/16SFWJ_1M7_0001.html.

\section{A viral RNA with a key alternate conformation}

Hepatitis Delta Virus (HDV) normally adopts a rod shaped configuration, but HDV Genotype III must also form a branched structure in order to undergo an essential RNA editing event [43]. We ran our MIBP algorithm on a section of the HDV Genotype III (reverse complement of GenBank: HF679406.1, nucleotides 499-1097). Most sampled structures form a rod shaped structure (Fig. 7a), but some form the branched structure described in [43] (Fig. 7b). The MIBP algorithm shows that the branched structure forms when the MIBP - $(1020,1086)$ is present and the rod structure forms when it is absent.

\section{SHAPE}

The MIBP algorithm can also be used to show how the inclusion of experimental data, such as SHAPE (selective 2'-hydroxyl acylation analyzed by primer extension) [44] affects the probability distribution. SHAPE data is used by RNAstructure to calculate a "pseudoenergy": $E^{*}(X)=$ $E(X)+C(X, D)$ where $C(X, D)$ is a "pseudoenergy change

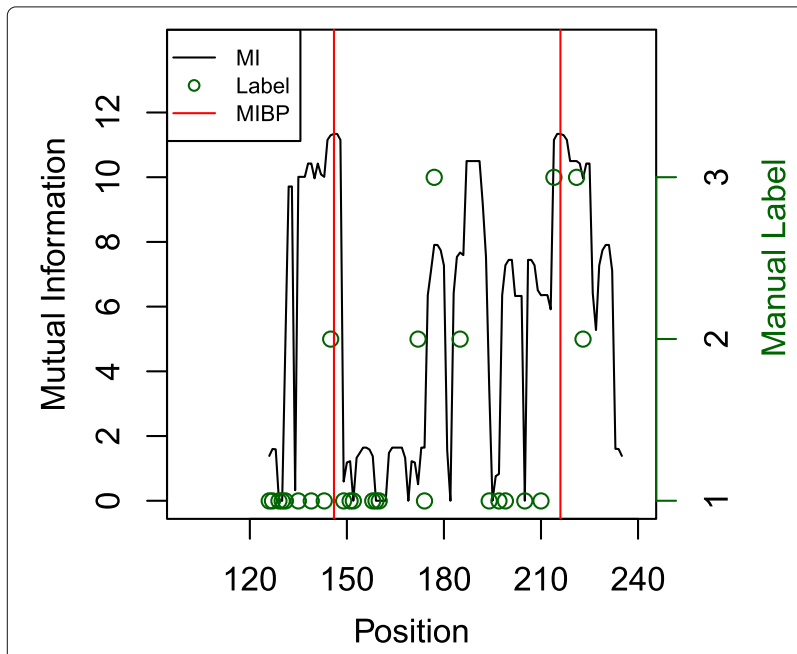

Fig. 6 Mutual information sum and label assigned by Woods and Laederach [42] for the 16s rRNA four way junction (16SFWJ_1M7_0001 in RMDB: https://rmdb.stanford.edu/). A label of 1 indicates that the mutation causes little or no change in RNA SS. A label of 2 indicates that the mutation causes significant but primarily local changes to the RNA SS. A label of 3 indicates that the mutation causes global changes to the RNA SS

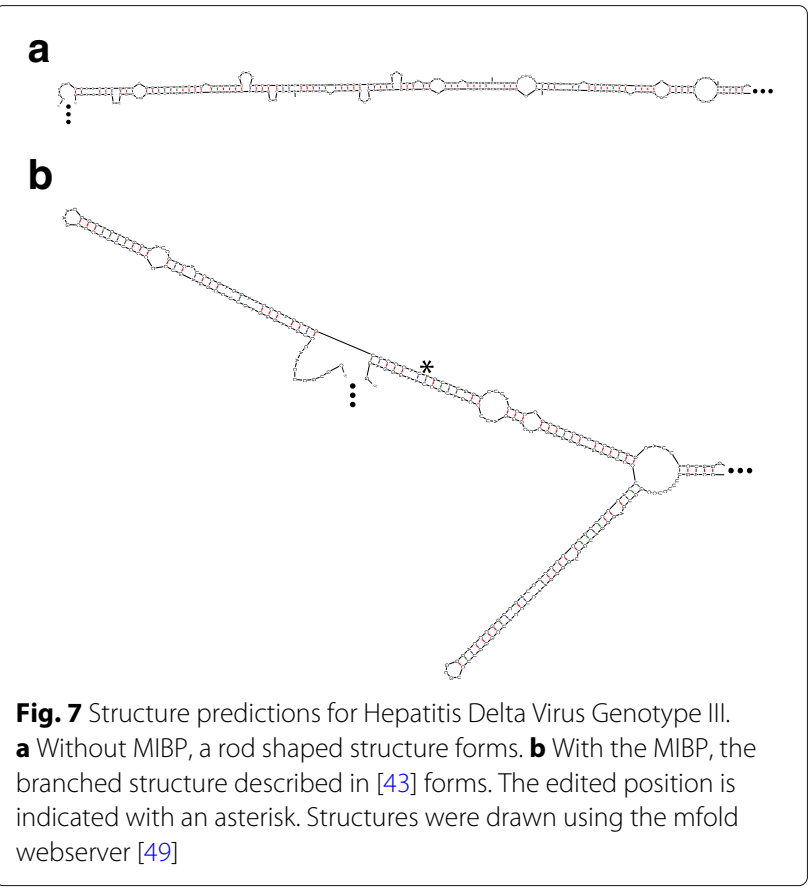

term" that reflects how well the structure $X$ fits the experimental data $D$ [45]. Since Boltzmann probability is calculated by exponentiating the free energy, this is equivalent to using the nearest neighbor model as a Bayesian prior and then updating it with a likelihood term calculated from the SHAPE data. The data we use is from [46] and [45].

The inclusion of SHAPE data yields a simpler distribution with fewer conflicting pairs and samples that are more similar to the native structure. When SHAPE data is included, entropy is lower by a factor ranging from 2 to 17.3. On average the expected difference between a sampled structure and the native structure measured in basepairs different decreases by a factor of 9.6 . Figure 8 shows a visualization of the distribution with and without SHAPE data for the most dramatic example - a phenylalanine tRNA. In two cases the inclusion of SHAPE yields a distribution in which no basepair has at least 2 bits of mutual information. For the other four sequences, the largest cluster contains the native structure. This is only true for two of the six sequences without SHAPE data "See Table 1".

\section{Discussion}

Our algorithm provides a characterization of the Boltzmann weighted space by iteratively dividing the secondary structure space based on the presence or absence of MIBPs. Mutual information allows us to find a small number of basepairs that account for about half of the uncertainty in the Boltzmann ensemble. We can then visualize the ensemble distribution as a finite mixture of a small set of simpler distributions. 


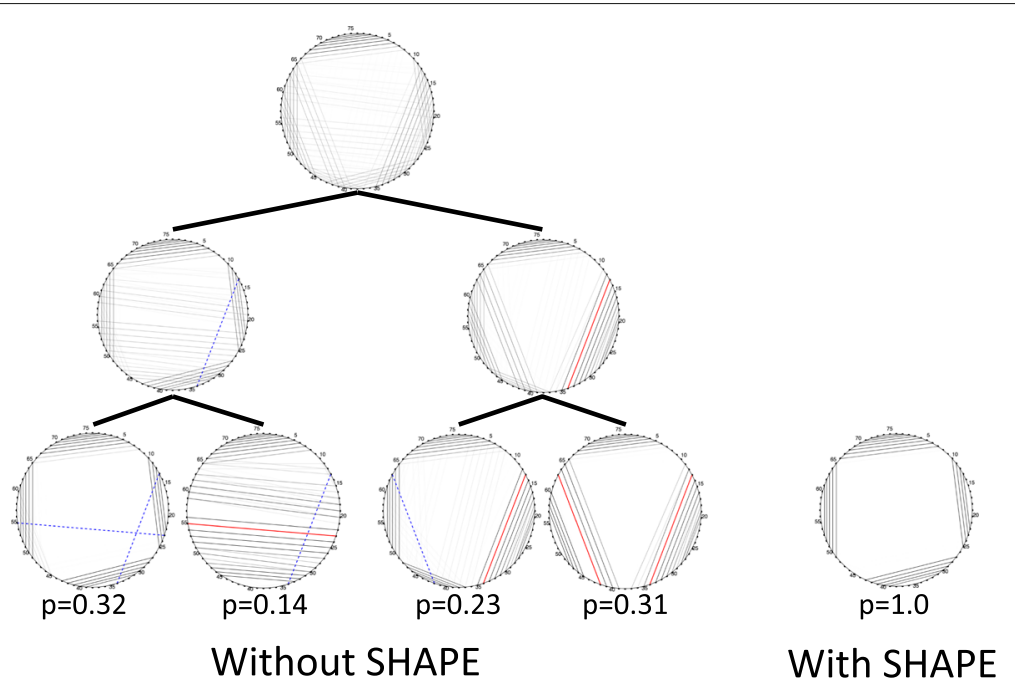

Fig. 8 Visualization of E. coli tRNA (Phe) Boltzmann space with and without SHAPE data. Nucleotides are arranged around the edge of a circle and basepairs are drawn as chords connecting the paired bases. MIBPs that are constrained to be present are highlighted in red. Those that are absent are highlighted in blue. The darkness of a plotted basepair is proportional to its probability. The native structure forms a clover-leaf shape as in the left most cluster and the cluster with SHAPE data

Our method differs from similar methods $[14,15,17,47]$ by focusing on the basepairs that cause the Boltzmann distribution to be multimodal. Our method not only groups similar structures, but also identifies the most informative basepairs that determine which group a structure falls into. The RNA profiling method [17] does provide a branching set of helices for each class. However using mutual information we are able to condense that set of

Table 1 Summary statistics for six sequences, with and without shape

\begin{tabular}{|c|c|c|c|c|c|}
\hline Sequence & Length & Clusters & Entropy & $\begin{array}{l}\text { Conditional } \\
\text { entropy }\end{array}$ & Mean error \\
\hline \multicolumn{6}{|c|}{ Without SHAPE data } \\
\hline Riboswitch & 71 & 3 & 25.3533 & 13.3428 & 5.5875 \\
\hline $5 s$ rRNA & 120 & 4 & 51.293 & 30.8405 & 53.3667 \\
\hline P546 & 155 & 7 & 114.5027 & 54.0569 & 44.1121 \\
\hline RNase $P$ & 154 & 8 & 86.7581 & 39.7549 & 36.8573 \\
\hline $\mathrm{HCV}$ & 95 & 5 & 37.893 & 11.3547 & 19.7718 \\
\hline tRNA (Phe) & 76 & 4 & 52.1349 & 17.404 & 20.474 \\
\hline \multicolumn{6}{|c|}{ With SHAPE data } \\
\hline Riboswitch & 71 & 1 & 4.9419 & 4.9419 & 1.1114 \\
\hline $5 s$ rRNA & 120 & 3 & 21.376 & 11.5638 & 10.1141 \\
\hline P546 & 155 & 3 & 28.1101 & 16.4472 & 7.1615 \\
\hline RNase $P$ & 154 & 3 & 20.3484 & 11.4831 & 20.9432 \\
\hline $\mathrm{HCV}$ & 95 & 2 & 18.6929 & 1.7029 & 6.4995 \\
\hline tRNA (Phe) & 76 & 1 & 3.0043 & 3.0043 & 0.5634 \\
\hline
\end{tabular}

Mean error is the expected number of basepairs that differ between the native structure and a sampled structure helices into a few key most informative basepairs. While two alternate structures may include very different sets of helices, it is often the case that one need only affect the stability of a single most informative basepair to bias one alternate structure over another.

With the realization that point mutations can change the secondary structure of an RNA transcript enough to cause misregulation and disease, there is a need to understand how SNPs affect RNA SS [18, 20]. MIBPs show that while alternate structures may have little overlap in shape or basepairing, it is often the case that constraining a single basepair is enough to bias one structure over another. Thus if a mutation disrupts the MIBP or its conflicting pair, it is likely to cause a global change in RNA SS. Indeed we find that mutations at positions with high mutual information are likely to have wide ranging effects on structure. Furthermore as new methods to edit specific sites in an RNA molecule emerge [48], the need for tools that can connect nucleotide changes to structure will only increase.

Finding MIBPs, provides valuable insight into the formation of alternate structures. The viral genome of HDV Genotype III must adopt an alternate conformation in order to undergo a key RNA editing event [43]. Our algorithm shows that the conformation can be predicted by the presence or absence of a single MIBP. This key basepair provide a starting place for understanding how and when this transition occurs.

Our results show that it only takes a few basepairs to encode half of the information present in a sample from the Boltzmann distribution. We also show that most possible basepairs have entropy near zero and are irrelevant to 
the Boltzmann distribution. If these low entropy pairs are constrained, the size of the structure space shrinks dramatically. Finally, characterizing the Boltzmann ensemble allows us to see how the incorporation of experimental data affects structure prediction.

The MIBP algorithm is not limited to the nearestneighbor thermodynamic model. Most informative basepairs exist for any probabilistic model of RNA and can be calculated either from samples or from the joint distributions of pairs of basepairs. In particular this strategy can be applied with ease to any single sequence stochastic context free grammar model. The MIBP algorithm uses single basepairs as its features, when it is generally whole helices and other large structural features that define an RNA secondary structure. In most cases a single MIBP can be used a proxy for a whole helix, but this can make finding conflicting basepairs difficult when two conflicting helices partially overlap. Nevertheless, mutual information is not limited to basepairs. Our algorithm could be combined with a method that abstracts from basepairs to find a most informative feature of some kind.

\section{Conclusion}

Most informative basepairs provide a novel method for exploring and visualizing the RNA secondary structure Boltzmann ensemble. Unlike other methods for characterizing the Boltzmann ensemble, the MIBP method provides a set of key basepairs that determine which structure will form from a given sequence. These pairs suggest that small changes either to the sequence or to the stability of specific pairs will bias a molecule to fold into one alternate structure over another.

\section{Additional methods}

Claim 1 For fixed $p_{i j}$, if $X_{i j}$ and $X_{k l}$ conflict, then $I\left(X_{i j} ; X_{k l}\right)$ is a monotonic increasing function of $p_{k l}$.

Proof: Using the formula for $I(X ; Y)$ given in [29] and the fact that $P\left(X_{i j}=1, X_{k l}=1\right)=0$ :

$$
\begin{aligned}
I\left(X_{i j} X_{k l}\right)= & \left(1-p_{i j}-p_{k l}\right) \log _{2} \frac{1-p_{i j}-p_{k l}}{\left(1-p_{i j}\right)\left(1-p_{k l}\right)} \\
& +p_{i j} \log _{2} \frac{p_{i j}}{p_{i j}\left(1-p_{k l}\right)}+p_{k l} \log _{2} \frac{p_{k l}}{p_{k l}\left(1-p_{i j}\right)} \\
= & \left(1-p_{i j}-p_{k l}\right) \log _{2}\left(1-p_{i j}-p_{k l}\right) \\
& -\left(1-p_{i j}\right) \log _{2}\left(1-p_{i j}\right)-\left(1-p_{k l}\right) \log _{2}\left(1-p_{k l}\right)
\end{aligned}
$$

Taking the derivative with respect of $p_{k l}$ yields:

$$
\begin{aligned}
\frac{d I\left(X_{i j} ; X_{k l}\right)}{d p_{k l}} & =\frac{1}{\log 2}\left[-2 \log \left(1-p_{i j}-p_{k l}+2 \log \left(1-p_{k l}\right)\right]\right. \\
& =\frac{2}{\log 2}\left[\log \frac{1-p_{k l}}{1-p_{i j}-p_{k l}}\right]>0
\end{aligned}
$$

Thus the claim is proved.

\section{Abbreviations}

HDV: Hepatitis Delta Virus; MIBP: most informative basepair; MFE: minimum free energy; RNAp: RNAse P; rRNA: ribosomal rna; SHAPE: selective 2'-hydroxyl acylation analyzed by primer extension; SRP: signal recognition particle; SS: secondary structure; tmRNA: transfer-messenger RNA; tRNA: transfer RNA

\section{Acknowledgements}

We would like to thank David Mathews for consulting on this project, for supplying test sequences, and for providing an easy way to count structures. We would also like to thank Matthew Harrison for his help in formulating the most informative basepair. Finally we would like to thank Alain Laederach for suggesting that we look at riboSNitches.

\section{Funding}

There were no funding sources dedicated to this research.

\section{Availability of data and materials}

Matlab implementation of the algorithm described here can be found at https://github.com/wmckerrow/MIBP. The raw RNA sequences considered can be found with the source code.

\section{Authors' contributions}

$\mathrm{LL}$ and $\mathrm{CL}$ formulated the most informative basepair. $\mathrm{LL}$ implemented algorithm 2. BR designed the visualization trees. WM and BR implemented algorithm 3. WM and CP generated results. WM wrote the paper. CL provided oversight and insight into the RNA SS model. All authors read and approved the final manuscript.

\section{Ethics approval and consent to participate}

No data was collected from people or animals of any kind.

\section{Consent for publication}

No personal data was reported.

\section{Competing interests}

The authors declare that they have no competing interests.

\section{Publisher's Note}

Springer Nature remains neutral with regard to jurisdictional claims in published maps and institutional affiliations.

\section{Author details}

${ }^{1}$ Center for Devices and Radiological Health, U.S. Food and Drug Administration, 20993 Silver Spring, MD, USA. ${ }^{2}$ Division of Applied Mathematics, Brown University, 02912 Providence, RI, USA. ${ }^{3}$ Software Engineer, Google, 10011 New York, NY, USA. ${ }^{4}$ Department of Mathematics, University of Southern California, 90089 Los Angeles, CA, USA.

Received: 15 September 2017 Accepted: 20 February 2018

Published online: 05 March 2018

\section{References}

1. Shapiro BA, Yingling YG, Kasprzak W, Bindewald E. Bridging the gap in RNA structure prediction. Curr Opin Struct Biol. 2007;17(2):157-65. https://doi.org/10.1016/j.sbi.2007.03.001.

2. Mathews DH. Revolutions in RNA secondary structure prediction. J Mol Biol. 2006;359(3):526-32. https://doi.org/10.1016/j.jmb.2006.01.067.

3. Bernhart SH, Hofacker IL, Will S, Gruber AR, Stadler PF. RNAalifold: improved consensus structure prediction for RNA alignments. BMC Bioinformatics. 2008;9(1):474. https://doi.org/10.1186/1471-2105-9-474

4. Xu Z, Mathews DH. Multilign: an algorithm to predict secondary structures conserved in multiple RNA sequences. Bioinformatics. 2011;27(5):626-32. https://doi.org/10.1093/bioinformatics/btq726.

5. Hofacker IL. Vienna RNA secondary structure server. Nucleic Acids Res. 2003;31(13):3429-31.

6. Reuter JS, Mathews DH. RNAstructure: software for RNA secondary structure prediction and analysis. BMC Bioinformatics. 2010;11:129. https://doi.org/10.1186/1471-2105-11-129.

7. Zuker M, Stiegler P. Optimal computer folding of large RNA sequences using thermodynamics and auxiliary information. Nucleic Acids Res. 1981;9(1):133-48. 
8. McCaskill JS. The equilibrium partition function and base pair binding probabilities for RNA secondary structure. Biopolymers. 1990;29(6-7): 1105-19. https://doi.org/10.1002/bip.360290621.

9. Ding $Y$, Lawrence CE. A statistical sampling algorithm for RNA secondary structure prediction secondary structure prediction. Nucleic Acids Res. 2003;31(24):7280-301. https://doi.org/10.1093/nar/gkg938.

10. Lu ZJ, Gloor JW, Mathews DH. Improved RNA secondary structure prediction by maximizing expected pair accuracy. RNA. 2009;15(10): 1805-13. https://doi.org/10.1261/rna.1643609.

11. Ding $Y$, Chan $C Y$, Lawrence CE. RNA secondary structure prediction by centroids in a boltzmann weighted ensemble. RNA. 2005;11(8):1157-66. https://doi.org/10.1261/rna.2500605.

12. Chan $C Y$, Lawrence $C E$, Ding Y. Structure clustering features on the Sfold web server. Bioinformatics. 2005;21(20):3926-8.

13. Ding $Y$, Chan CY, Lawrence CE. Clustering of RNA secondary structures with application to messenger RNAs. J Mol Biol. 2006;359(3):554-71. https://doi.org/10.1016/j.jmb.2006.01.056.

14. Giegerich R, Voß B, Rehmsmeier M. Abstract shapes of RNA. Nucleic Acids Res. 2004;32(16):4843-51. https://doi.org/10.1093/nar/gkh779.

15. Steffen P, VoßB, Rehmsmeier M, Reeder J, Giegerich R. RNAshapes: an integrated RNA analysis package based on abstract shapes. Bioinformatics. 2006;22(4):500-3.

16. Janssen S, Giegerich R. Faster computation of exact RNA shape probabilities. Bioinformatics. 2010;26(5):632-9. https://doi.org/10.1093/ bioinformatics/bta014.

17. Rogers $E$, Heitsch CE. Profiling small RNA reveals multimodal substructural signals in a Boltzmann ensemble. Nucleic Acids Res. 2014;42(22):171. https://doi.org/10.1093/nar/gku959.

18. Halvorsen M, Martin JS, Broadaway S, Laederach A. Disease-associated mutations that alter the RNA structural ensemble. PLoS Genet. 2010;6(8): 1001074. https://doi.org/10.1371/journal.pgen.1001074.

19. Wan Y, Qu K, Zhang QC, Flynn RA, Manor O, Ouyang Z, Zhang J, Spitale RC, Snyder MP, Segal E. Landscape and variation of RNA secondary structure across the human transcriptome. Nature. 2014;505(7485):706-70900280836.

20. Solem AC, Halvorsen M, Ramos SBV, Laederach A. The potential of the ribosnitch in personalized medicine. Wiley Interdiscip Rev RNA. 2015;6(5): 517-32. https://doi.org/10.1002/wrna.1291.

21. Ritz J, Martin JS, Laederach A. Evaluating our ability to predict the structural disruption of RNA by SNPs. BMC Genomics. 2012;13(Suppl 4):6. https://doi.org/10.1186/1471-2164-13-S4-S6.

22. Simon $A E$, Gehrke L. RNA conformational changes in the life cycles of RNA viruses, viroids, and virus-associated RNAs. Biochim Biophys Acta. 2009;1789(9-10):571-83. https://doi.org/10.1016/j.bbagrm.2009.05.005.

23. Thirumalai D, Lee N, Woodson SA, Klimov D. Early event in RNA folding Annu Rev Phys Chem. 2001;52(1):751-62. https://doi.org/10.1146/ annurev.physchem.52.1.751.

24. Sükösd Z, Knudsen B, Anderson JW, Novák Á, Kjems J, Pedersen CN. Characterising RNA secondary structure space using information entropy. BMC Bioinformatics. 2013;14(2):22. https://doi.org/10.1186/1471-210514-S2-S22.

25. Manzourolajdad A, Arnold J. Secondary structural entropy in RNA switch (riboswitch) identification. BMC Bioinformatics. 2015;16:133. https://doi.org/10.1186/s12859-015-0523-2.

26. Schneider TD, Stormo GD, Gold L, Ehrenfeucht A. Information content of binding sites on nucleotide sequences. J Mol Biol. 1986;188(3):415-31. https://doi.org/10.1016/0022-2836(86)90165-8.

27. Gutell RR, Power A, Hertz GZ, Putz EJ, Stormo GD. Identifying constraints on the higher-order structure of RNA: continued development and application of comparative sequence analysis methods. Nucleic Acids Res. 1992;20(21):5785-95.

28. Mathews DH, Disney MD, Childs JL, Schroeder SJ, Zuker M, Turner DH. Incorporating chemical modification constraints into a dynamic programming algorithm for prediction of RNA secondary structure. Proc Natl Acad Sci U S A. 2004;101(19):7287-92. https://doi.org/10.1073/pnas 0401799101.

29. Cover T, Thomas J. Elements of Information Theory. Hoboken: Wiley; 1991.

30. Quinlan JR. Induction of decision trees. Mach Learn. 1986;1(1):81-106. https://doi.org/10.1023/A:1022643204877.

31. Szymanski M, Specht T, Barciszewska MZ, Barciszewski J, Erdmann VA. 5s rRNA data bank. Nucleic Acids Res. 1998;26(1):156-59.
32. Gutell RR, Gray MW, Schnare MN. A compilation of large subunit (23s and 23s-like) ribosomal RNA structures. Nucleic Acids Res. 1993;21(13): 3055-74.

33. Gutell RR. Collection of small subunit (16s- and 16s-like) ribosomal RNA structures. Nucleic Acids Res. 1994;22(17):3502-7.

34. Damberger $\mathrm{SH}$, Gutell RR. A comparative database of group I intron structures. Nucleic Acids Res. 1994;22(17):3508-10.

35. Michel F, Kazuhiko U, Haruo O. Comparative and functional anatomy of group II catalytic introns - a review. Gene. 1989;82(1):5-30. https://doi. org/10.1016/0378-1119(89)90026-7.

36. Brown JW, Haas ES, Gilbert DG, Pace NR. The Ribonuclease P database. Nucleic Acids Res. 1994;22(17):3660-2.

37. Larsen N, Zwieb C. The signal recognition particle database (SRPDB). Nucleic Acids Res. 1996;24(1):80-1.

38. Podlevsky JD, Bley CJ, Omana RV, Qi X, Chen JJ-L. The telomerase database. Nucleic Acids Res. 2008;36(Database issue):339-43. https://doi. org/10.1093/nar/gkm700.

39. Zwieb C, Gorodkin J, Knudsen B, Burks J, Wower J. tmRDB (tmRNA database). Nucleic Acids Res. 2003;31(1):446-7.

40. Sprinzl M, Vassilenko KS. Compilation of tRNA sequences and sequences of tRNA genes. Nucleic Acids Res. 2005;33(Database Issue):139-40. https://doi.org/10.1093/nar/gki012.

41. Talagrand M. A new look at independence. Ann Probab. 1996;24(1):1-34.

42. Woods CT, Laederach A. Classification of rna structure change by 'gazing'at experimental data. Bioinformatics. 2017;33(11):1647-55. https://doi.org/10.1093/bioinformatics/btx041.

43. Casey JL. RNA editing in hepatitis delta virus genotype III requires a branched double-hairpin rna structure. J Virol. 2002;76(15):7385-97. https://doi.org/10.1128/JVI.76.15.7385-7397.2002.

44. Merino EJ, Wilkinson KA, Coughlan JL, Weeks KM. RNA structure analysis at single nucleotide resolution by Selective 2'-Hydroxyl Acylation and Primer Extension (SHAPE). J Am Chem Soc. 2005;127(12):4223-31. https://doi.org/10.1021/ja043822v.

45. Deigan KE, Li TW, Mathews DH, Weeks KM. Accurate SHAPE-directed RNA structure determination. Proc Natl Acad Sci U S A. 2009;106(1): 97-102. https://doi.org/10.1073/pnas.0806929106.

46. Leonard CW, Hajdin CE, Karabiber F, Mathews DH, Favorov O, Dokholyan NV, Weeks KM. Principles for understanding the accuracy of SHAPE-directed RNA structure modeling. Biochemistry. 2013;52(4): 588-95. https://doi.org/10.1021/bi300755u.

47. Freyhult E, Moulton V, Clote P. RNAbor: a web server for RNA structural neighbors. Nucleic Acids Res. 2007;35(Web Server issue):305-9. https://doi.org/10.1093/nar/gkm255.

48. Cox DBT, Gootenberg JS, Abudayyeh OO, Franklin B, Kellner MJ, Joung J, Zhang F. RNA editing with CRISPR-Cas13. Science. 2017;358:1019-27.

49. Zuker M. Mfold web server for nucleic acid folding and hybridization prediction. Nucleic Acids Res. 2003;31(13):3406-15.

\section{Submit your next manuscript to BioMed Central and we will help you at every step:}

- We accept pre-submission inquiries

- Our selector tool helps you to find the most relevant journal

- We provide round the clock customer support

- Convenient online submission

- Thorough peer review

- Inclusion in PubMed and all major indexing services

- Maximum visibility for your research

Submit your manuscript at www.biomedcentral.com/submit
( Biomed Central 